\title{
BuMNercology open Driving restrictions following deep brain stimulation surgery
}

\author{
Andrew Roy Charmley (D) , ${ }^{1}$ Thomas Kimber, ${ }^{2,3}$ Neil Mahant, ${ }^{4}$ Alexander Lehn ${ }^{1,5}$
}

To cite: Charmley AR, Kimber T, Mahant N, et al. Driving restrictions following deep brain stimulation surgery. BMJ Neurology Open 2021;3:e000210. doi:10.1136/ bmjno-2021-000210

Received 08 August 2021 Accepted 28 October 2021

\section{Check for updates}

C Author(s) (or their employer(s)) 2021. Re-use permitted under CC BY-NC. No commercial re-use. See rights and permissions. Published by BMJ.

${ }^{1}$ Department of Neurology, Princess Alexandra Hospital, Woolloongabba, Queensland, Australia

${ }^{2}$ Central Adelaide Neurology Service, Royal Adelaide Hospital, Adelaide, South Australia, Australia

${ }^{3}$ Department of Medicine, University of Adelaide, Adelaide, South Australia, Australia ${ }^{4}$ Department of Neurology, Westmead Hospital, Westmead, New South Wales, Australia ${ }^{5}$ University of Queensland, Brisbane, Queensland, Australia

Correspondence to

Dr Alexander Lehn;

a.lehn@uq.edu.au

\begin{abstract}
Background There are currently no Australian guidelines to assist clinicians performing deep brain stimulation (DBS) procedures in setting postoperative driving restrictions.

Purpose We aimed to provide recommendations for postDBS driving restrictions to guide practice in Australia. Methods A review of current Australian and international driving guidelines, literature regarding the adverse effects of DBS and literature regarding the long-term effect of neurostimulation on driving was conducted using Elton $B$ Stephens Company discovery service-linked databases. Australian neurologists and neurosurgeons who perform DBS were surveyed to gain insight into existing practice.

Results No guidance on driving restrictions following DBS surgery was found, either in existing driving guidelines or in the literature. There was a wide difference seen in the rates of reported adverse effects from DBS surgery. The most serious adverse events (haemorrhage, seizure and neurological dysfunction) were uncommon. Longer term, there does not appear to be any adverse effect of DBS on driving ability. Survey of Australian practitioners revealed a universal acceptance of the need for and use of driving restrictions after DBS but significant heterogeneity in how return to driving is managed.

Conclusion We propose a 6-week driving restriction for private licences and 6-month driving restriction for commercial licences in uncomplicated DBS. We also highlight some of the potential pitfalls and pearls to assist clinicians to modify these recommendations where needed. Ultimately, we hope this will stimulate further examination of this issue in research and by regulatory bodies to provide more robust direction for practitioners performing DBS implantation.
\end{abstract}

\section{INTRODUCTION}

Deep brain stimulation (DBS) surgery is a useful tool in the treatment of movement disorders (such as Parkinson's disease (PD), tremor and dystonia), although it is increasingly used and/or investigated for other conditions such as epilepsy and chronic pain. It is important that a practitioner understands the risks and limitations of this technology. This is especially true in regard to the driving task, as both a key aspect of quality of life for people undergoing this therapy and in the broader societal implications of driving safety.

There is currently no Australian guideline on what driving restrictions should be placed on patients undergoing DBS surgery, and there is a paucity of evidence related to risks that DBS poses to driving. Thus, it is up to individual units to specify arbitrary driving restrictions, exposing DBS teams to potential medicolegal consequences should a driving incident occur.

Herein, we aimed to assist in making decisions about driving restrictions relating to DBS. It will examine (1) relevant current Australian driving guidelines and international driving guidelines, (2) the current evidence related to postoperative and longterm risks of DBS that may pertain to driving, (3) the impact of neurostimulation long term on driving, and (4) the currently used restrictions and consensus opinion of Movement Disorder Neurologists preforming DBS in Australia.

\section{METHODS}

A search using Elton B Stephens Company (EBSCO) discovery service-linked databases (via the Clinicians Knowledge Network) and Google was performed to look for current driving guidelines. The term 'driving guideline', combined with tems from Englishspeaking countries (ie, Canada and New Zealand), was used. Only official government (local or national) guidelines were included in this study.

To examine the adverse effects of DBS surgery, a search using EBSCO discovery service-linked databases (via the Clinicians Knowledge Network) was performed using the terms "DBS", "deep brain simulation", "side effects", "adverse effects", "seizure", "stroke", "haemorrhage" and "neuropsy*". Only original research articles published in English were included in the study. All articles were reviewed by AC. References from included articles were searched for other relevant articles.

Studies of the effect of neurostimulation on driving were identified by searching the terms; 'DBS' 'deep brain stimulation', 'driving', 'drive', and 'motor vehicle' using 
EBSCO discovery service linked databases (via the Clinicians Knowledge Network). Articles in English were reviewed by ARC for inclusion. References from included articles were searched for other relevant articles.

A nine-question survey was devised by ARC, AL, TC and SM and was mailed to Australian neurologists and neurosurgeons from the contacts of AL, TC and SM. Respondents were encouraged to forward the survey to other practitioners performing DBS surgery not included in the initial list. A remainder was emailed 2 weeks later. Responses were collected from January to December 2020.

\section{FINDINGS}

\section{Current national and international driving guidelines}

We could not identify specific recommendations regarding driving following DBS surgery in any local jurisdiction. In particular, the current Austroads guidelines provide no specific guidance of post-DBS driving restrictions. The UK is the only driving guideline reviewed with a recommendation for driving after DBS: for a private licence, they allow resumption of driving, providing there are no complications of the surgery; the patient is seizurefree; and there are no debarring residual impairments likely to affect safe driving. ${ }^{1}$ However, the timing of return to driving is not specified.

Austroads provides an advisory regarding intracranial surgery that suggests that a patient undergoing supratentorial surgery should have a driving restriction (6 months for a standard private licence and 12 months for a commercial licence) to account for the risk of seizure. As this is an advisory only, non-driving periods may be modified by the surgeon. ${ }^{2}$ No further detail regarding specific surgeries is provided. No recommendations are made for DBS or catheter-based neurosurgery such as insertion of a ventricular catheter for pressure monitoring or cerbrospinal fluid diversion.

Advice for non-DBS neurosurgery varies by indication and jurisdiction. The National Highway Traffic Safety Administration guideline of the $\mathrm{USA}^{3}$ does not give specific guidance on returning to driving after neurosurgery. The UK Driver and Vehicle Licensing Agency $^{1}$ advises a 6-month driving restriction in patients undergoing supratentorial craniotomy, but this may be higher for malignant indications. Burr hole surgery does not carry a required non-driving period for either intracranial pressure monitoring or DBS insertion. ${ }^{1}$ Canadian guidelines have guidance only for epilepsy surgery (6-month seizure-free for a private licence) and aneurysm repair (3 months for a private licence and 6 months for a commercial licence). ${ }^{4}$ New Zealand Transport Agency guidelines require a 12-month driving-free period after treating an intracranial tumour (with any modality including surgery), ${ }^{5}$ extended to 3 years in advanced or metastatic tumours and decreased to 6 months for pituitary tumours or aneurysms treated with craniotomy. ${ }^{5}$
Regarding PD, the current Austroads guideline states that driving performance may be affected by motor and cognitive manifestations of PD. It also highlights the need to consider the impact of motor fluctuations on driving ability. ${ }^{2}$ Criteria by which to assess the driver's ability are not specified, nor criteria for referral for an on-road driving assessment.

International driving guidelines regarding driving with PD are similar to the Austroads guideline in highlighting the potential impact of PD on driving performance, particularly motor fluctuations. ${ }^{134}$ The guidelines support the use of functional assessments to assist in deciding capacity to drive, though there is little specific guidance given on when, or in whom, assessments or suspension of driving licence should be considered. ${ }^{13-5}$

\section{Postoperative risks of DBS}

Uncomplicated DBS surgery may have little impact on the ability to drive, which may be primarily affected by complications of surgery. Several studies have examined the rates of adverse events following DBS insertion, with a wide range of the rate of adverse events $(4.2 \%-37 \%$, table 1). ${ }^{6-15}$ These studies primarily examine the risks of DBS in movement disorders (PD, tremor and dystonia) as the most common indication. However, a number of studies examined the risks of DBS in other indications such as Gilles de Tourette's syndrome, epilepsy, obsessive compulsive disorder and chronic pain. ${ }^{912} 14$ Risks following DBS include intracerebral haemorrhage (ICH), stroke, transient neurological dysfunction, infections and hardware issues with the DBS system. ${ }^{6-15}$

One potentially devastating adverse event of DBS insertion is $\mathrm{ICH}$, with rates reported between $0.4 \%$ and $5.0 \%$ at 30 days of follow-up, and symptomatic ICH between $0 \%$ and $1.8 \%$. The risk of ICH is highest intraoperatively or immediately postoperatively (within the first 24 hours). ${ }^{12-14}$ Late haemorrhage generally occurred due to factors independent from or in addition to the DBS. ${ }^{15}$ Careful electrode trajectory planning to avoid structures that may bleed (eg, vessels, the deep sulci and the highly vascularised periventricular space) might reduce the risk of ICH. ${ }^{9} 16$ The DBS target selected (globus pallidus), a diagnosis of $\mathrm{PD}$, advanced age, hypertension and medical comorbidities have been reported to increase the risk of ICH. The data for this are, however, conflicting and inconsistent. ${ }^{101117}$ Ischaemic stroke following DBS appears rare $(0 \%-0.4 \%)$ and may be related to underlying factors such as coagulopathy. ${ }^{15}$ Neurological deficits due to ICH or ischaemic stroke could adversely affect driving ability, and driving restrictions will depend on the nature and severity of these issues rather than DBS surgery per se. The presence of a stroke and the accompanying neurological deficits will generally be identified prior to discharge from the hospital, and therefore the risk of stroke should not necessarily have a direct impact on driving recommendations for uncomplicated DBS surgery.

Seizure following DBS has been reported to affect between $0 \%$ and $3 \%$ of DBS surgeries. The risk of seizure 


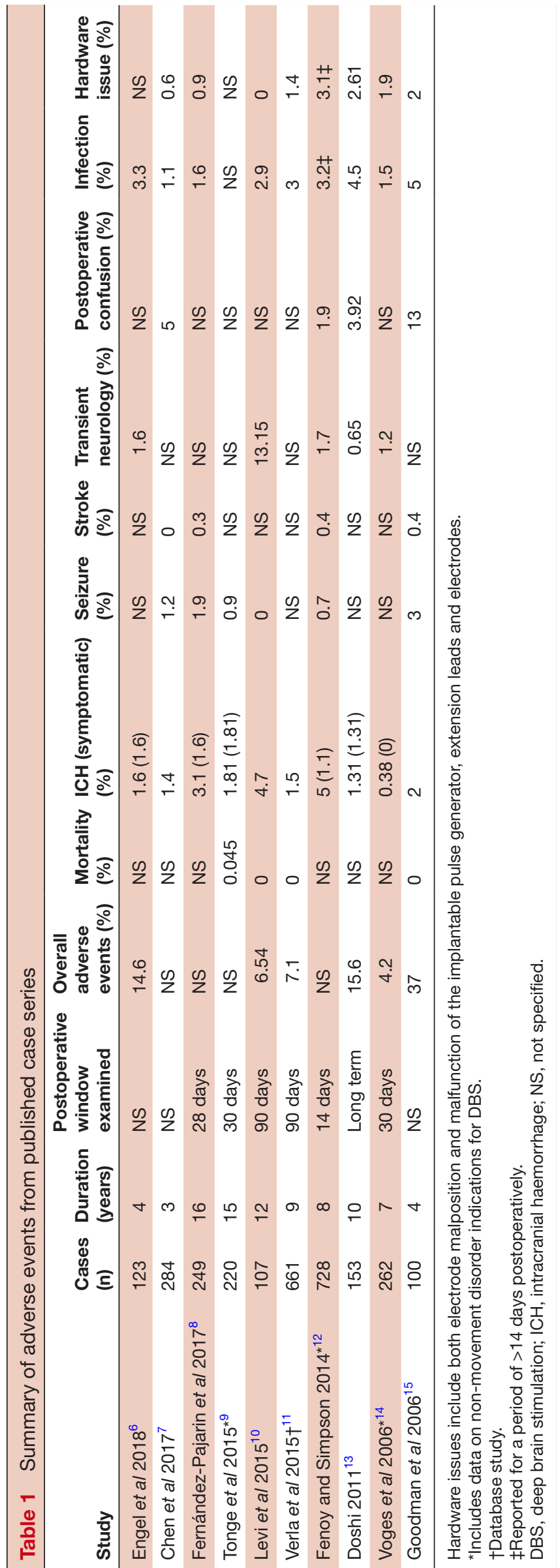

is highest either intraoperatively or in the immediate postoperative period. ${ }^{71218}$ Seizures occurring outside of the perioperative window accounted for $<25 \%$ of seizures, reportedly occurring after $0.5 \%$ of DBS surgeries. ${ }^{18}$ The risk of recurrent seizures appears low, though there are minimal data available on this outcome. ${ }^{9}$ Risk factors for seizure are not well reported with ICH, oedema and ischaemia being reported. ${ }^{18} 19$

Perielectrode oedema may cause focal neurological signs. It may be caused by an inflammatory reaction to the insertion of the electrode rather than ischaemia, ${ }^{8}$ with an estimated prevalence of $0.7 \%-13.5 \%$ of surgeries. Neurological dysfunction is generally self-limiting over weeks to months, and the presence of focal neurological deficits would mandate modification of driving restrictions. Perielectrode oedema may also result from bacterial infection spreading along the brain lead and may be difficult to treat without surgical removal of the infected lead. Infection is a serious consequence of DBS insertion, occurring acutely after implantation surgery in $1 \%-5 \%$ of cases, typically at the implantable pulse generator pocket, but may occur at any point of the DBS system. ${ }^{78}$ If the infection cannot be controlled with antibiotics, then the infected component of the DBS hardware requires removal. ${ }^{15}$ Infection may occur at any time following implantation of the DBS system. ${ }^{81215}$

Transient postoperative delirium (POD) is common, occurring in up to $13 \%$ of cases. Risk factors for POD are best defined for people with PD and include a history of delirium, age, disease duration, white matter volume and cerebral oedema. ${ }^{2021} \mathrm{POD}$ is generally short-lived, with an average duration of 1.5 days, with a maximum duration of 13 days in one sample, ${ }^{22}$ which agrees with our clinical experience. We would recommend that the treating clinician ensure that POD has fully resolved prior to allowing a return to driving, taking into account that mild delirium may be difficult to diagnose in a hospital environment.

Neuropsychiatric complications of DBS also have the potential to affect driving performance. Neuropsychiatric side effects include severe depression, hypomania/mania and suicide. ${ }^{23}$ There is also good evidence for impaired executive function with subthalamic nucleus (STN) DBS. ${ }^{23}{ }^{24}$ The occurrence of neuropsychiatric symptoms appears to be influenced by DBS target, stimulation parameters, other medications and electrode tract. ${ }^{23}$ The incidence appears highest with STN DBS, higher stimulation parameters, increased doses of dopaminergic medication and tracts crossing the lateral ventricular walls. ${ }^{23}$ Neuropsychiatric symptoms often only become apparent after a delay of hours to days-after the patient has left the clinical environment. The impact of neuropsychiatric effects on driving is not documented. There exist reports of motor vehicle crashes that have been attributed to frontal disinhibition from DBS, ${ }^{25}$ but this is not backed by robust examination of this issue. Nevertheless, we would recommend great care in examining for neuropsychiatric adverse effects of DBS when considering return to driving after DBS. 


\section{Effect of long-term neurostimulation on driving}

Little data are available on the long-term effect of DBS on the driving task. In one survey of people with PD, it was found that about $50 \%$ of patients are driving following DBS insertion, ${ }^{26}$ which is similar to the driving rate in unselected patients with PD. Most patients reported that they did not drive due to the severity of PD-related symptoms. ${ }^{26}$ Following DBS, patients who were driving were younger, had less severe disease, had a shorter duration of DBS, were less cognitively impaired (higher MiniMental State Exam scores) and were more likely to be male. ${ }^{26}$

It is possible the DBS might allow people with PD to drive longer than otherwise might have been possible. Following DBS insertion, 23\% of patients who did not drive prior to surgery resumed driving within 12 months, while only $11 \%$ of patients who drove prior to the surgery ceased driving in the same period. ${ }^{26}$ There was no difference between selfreported motor vehicle crashes in the 3 months prior to DBS insertion compared with the 12 months after. ${ }^{26}$ However, it should be noted that the survey was done retrospectively, and so the results may be affected by recall or selection bias. In one study, people with PD treated with STN DBS had significantly fewer slight, severe and very severe errors when compared with people with PD not treated with DBS. ${ }^{27}$ When compared with non-PD controls, the DBS patients did not demonstrate an increased rate of errors. ${ }^{26}$ The same group examined the effect of STN DBS versus levodopa. Despite similar motor dysfunction as measured by the Unified Parkinson Disease Rating Scale III, there were fewer errors in the DBS only compared with the levodopa-only state, and the levodopaonly state did not differ significantly from the no-treatment state in terms of error rate. ${ }^{26}$ They speculated that improved sequential learning and goal-orientated activity with STN DBS may lead to better decision making and better attention to the driving task in DBS patients. Additionally, the reduction in dopaminergic medications afforded by STN DBS may contribute to reduced impulsiveness, improving decision making. ${ }^{26}$

Another potential benefit of STN DBS in PD is a reduction in daytime sleepiness. A recent meta-analysis of sleep in STN DBS showed improvements in subjective sleep measures. The effect was modest and not persistent for all measures with Epworth sleepiness scores returning to baseline after 12 months. ${ }^{28}$ Polysomnographic studies demonstrated a decreased rapid eye movement sleep latency but no other objective changes in sleep architecture or efficiency. ${ }^{28}$ Some of these effects are likely due to the medication reduction afforded by STN DBS as well as improvement in motor symptoms. ${ }^{28}$

More important than the possibility of improved driving following DBS seen in these studies, there was no danger signal seen. ${ }^{27}$ Though larger and more inclusive studies would be ideal to answer the question of the effect of DBS on long-term driving performance, the current evidence would suggest that, for STN DBS at least, there is no specific danger over that seen in non-DBS sufferers with PD.
There are no available data concerning the effects of DBS at other targets on driving, or the effects of STN DBS for conditions other than PD.

\section{Effect of underlying condition on driving}

When deciding driving restrictions following DBS, it is also important to consider the impact of the underlying condition on driving performance. PD is the best studied indication for DBS treatment, with respect to its effect on driving. Factors affecting driving performance in PD include motor and non-motor disease manifestations. Motor symptoms have demonstrated detrimental effects on reaction time and steering accuracy. ${ }^{29}$ The cognitive dysfunction associated with PD is likely have the greatest impact on driving performance. ${ }^{29}{ }^{30}$ Poor visual processing, particularly in non-ideal contrast and lighting, also accounts for some of the increased risk. ${ }^{29}$ Finally, excessive daytime sleepiness is a prominent feature in PD sufferers and may be particularly exacerbated by dopaminergic medication use. ${ }^{29}$

There are less data available on the effect of tremor and dystonia on driving. Studies in cervical dystonia did not detect a difference from the control group in a driving simulator. ${ }^{31}$ No data pertaining to driving with tremor could be found. In both of these conditions, motor, nonmotor and cognitive symptoms may be less severe than PD and therefore have less impact on driving performance.

\section{Currently used driving restrictions in Australia}

A nine-question survey was sent to 35 neurologists and neurosurgeons in Australia performing DBS surgery. The survey was returned by 15 respondents to the survey, 11 of whom (73\%) were neurologists. All respondents indicated that they applied driving restrictions following routine DBS surgery, with $67 \%$ indicating that driving restrictions are discussed prior to surgery only, $13 \%$ after surgery only, and $20 \%$ both before and after surgery. The recommendations were given verbally by $47 \%$, with the remainder providing both verbal and written recommendations. No respondents provided written recommendations only. All respondents indicated that they used general restrictions in uncomplicated DBS and did not consider indication-specific driving restrictions.

For private licences, driving restrictions ranged from 4 weeks to 3 months. Driving restrictions for commercial licences were not as well documented, with nine respondents indicating they did not have specific recommendations or had not encountered this situation. One respondent indicated that cases were individually assessed, and one respondent used occupational therapy driving assessment prior to return to driving, others recommending a restriction of 3-6 months.

Most respondents indicated that they would modify their driving restrictions based on other considerations. Factors considered can be divided into complications (delirium, seizure and neurological deficit), underlying disease characteristics (preoperative performance, physical disability, impulsivity and insight) and therapy-related factors (response to therapy). 


\section{DISCUSSION}

Local and international guidelines provide little clarity regarding the need for and duration of driving restrictions following DBS surgery. Taken together, the current evidence suggests that post-DBS surgery driving restrictions are warranted, but the duration of this restriction varies between centres in Australia. This variability reflects a lack of data to inform clinicians. Let us consider which factors could have an impact on the ability to drive following DBS surgery. Most of the risks of surgery become apparent immediately or within days of surgery. Outside of this, potentially the most important issues in terms of return to driving are undiagnosed delirium, undiagnosed focal neurological deficits (such as visuospatial neglect) and the onset of seizures days to weeks after surgery. All these factors would be expected to be rare even 2 weeks after DBS surgery, and therefore, it is reasonable to prescribe abstinence from driving for several weeks following surgery. One surgical complication that often has a delay prior to symptomatic onset is perielectrode oedema, with the possibility of causing neurological deficits days to a couple of weeks after surgery, by which time the patient may have returned home and is no longer under close observation by the treating team. This carries the potential for significant neurological deficits to become manifest and affect driving ability. Overall, the risk of new adverse events related to the DBS surgery would appear to be low after the first postoperative month.

Currently available data suggest that long-term STN DBS for PD does not negatively affect driving ability. This would suggest that the presence of a DBS system does not mandate specific restrictions on driving.

The main limitation in the current data is the lack of studies and guidelines specifically addressing the effect of DBS on driving. Though adverse effect rates following DBS surgery are well documented, the specific impact of this on driving post-DBS is not explicitly addressed in current studies. Furthermore, there are very little data on the long-term effects of neurostimulation on driving, and only in specific DBS indications.

\section{CONCLUSION}

We propose a 6-week driving restriction for private licence holders following DBS surgery with the following caveats: (1) the surgery and postoperative recovery is uncomplicated; (2) the underlying disease is sufficiently controlled and presents no contraindication to driving; and (3) there are no adverse effects from surgery or neurostimulation that might impair the ability to drive. We would recommend obtaining information, not only from the members of the treating team but also from family members of patients undergoing DBS who may be better able to identify more subtle or delayed adverse effects. Driving restrictions may need to be extended if any of the aforementioned criteria are not met. The last criterion is difficult because of the breadth of potential neurological and neuropsychiatric issues than can occur following DBS surgery, many of which may not be identified during a routine neurological assessment. This recommendation is made in consideration of (1) low risk of adverse events, specifically seizure, after the first postoperative month; (2) local and international driving guidelines recommending driving restrictions of 3-6 months following neurosurgery; (3) lack of evidence for negative effects of neurostimulation on the driving task in the absence of adverse effects from the neurostimulation; and (4) author consensus taking into consideration local and personal practices.

We propose a 6-month driving restriction for commercial licence holders following DBS with the following restrictions: (1) the surgery and postoperative recovery are uncomplicated; (2) the underlying disease is sufficiently controlled and presents no contraindication to driving; (3) there are no adverse effects from neurostimulation that pose a contraindication to driving; and (4) neurological, neurosurgical, neuropsychiatric and neuropsychological assessments are undertaken prior to resumption of commercial driving. We recommend individualising restrictions where these conditions are not met. Occupational therapy-guided driving assessments are recommended where there is any concern regarding driving performance. This recommendation is made in consideration of (1) greater consequences of traffic crashes inherent in commercial driving tasks; (2) low risk of adverse events, specifically seizure, after the first postoperative month; (3) lack of clear guidance for commercial licence holders in local and international driving guidelines; (4) lack of evidence for negative effects of neurostimulation on the driving task in the absence of adverse effects from the neurostimulation; and (5) author consensus taking into consideration local and personal practices.

It should be noted that the presence of a DBS system does not constitute the only risk to driving, and it is important to consider the effect of the underlying indication for the DBS when advising on return to driving.

Data-driven recommendations are needed, and this will require prospective studies including driving-related issues, and of laboratory or clinic-based paradigms relevant to driving tasks. However, these studies are difficult because of the need for a large sample size when examining rare outcomes, and the complexity of extrapolating from a laboratory paradigm to real-world driving. In the absence of such data, we must rely on clinical experience and expert opinion, as summarised previously.

\section{Acknowledgements The authors acknowledge all the neurologists and neurosurgeons who responded to the survey for their contribution to building a picture of deep brain stimulation driving restrictions currently in use across Australia.}

Contributors ARC was the primary author of this paper. AL, NM and TK were cocontributors in editing the paper and deciding the recommendations put forth.

Funding The authors have not declared a specific grant for this research from any funding agency in the public, commercial or not-for-profit sectors.

Competing interests ARC was participating in a movement disorders fellowship in 2019 partially funded by Abbott medical. TK had paid travel to DBS workshop (DBS user group meeting) paid for by Medtronic. NM reports no conflicts of interest. AL reports no conflicts of interest. This article was written by the authors with no input from any outside entity. The editing, recommendations and decision to published were not influenced by any external entity.

Patient consent for publication Not applicable. 
Provenance and peer review Not commissioned; internally peer reviewed.

Open access This is an open access article distributed in accordance with the Creative Commons Attribution Non Commercial (CC BY-NC 4.0) license, which permits others to distribute, remix, adapt, build upon this work non-commercially, and license their derivative works on different terms, provided the original work is properly cited, appropriate credit is given, any changes made indicated, and the use is non-commercial. See: http://creativecommons.org/licenses/by-nc/4.0/.

ORCID iD

Andrew Roy Charmley http://orcid.org/0000-0002-8939-8259

\section{REFERENCES}

1 Driver and vehicle licensing agency. Assessing fitness to drive - a guide for medical professionals. United Kingdom, 2019. Available: www.gov.uk/dvla/fitnesstodrive [Accessed 5 Nov 2019].

2 National transport commission. Assessing fitness to drive for commercial and private vehicle drivers. Australia, 2016. Available: https://austroads.com.au/_data/assets/pdf_file/0022/104197/AP_ G56-17_Assessing_fitness_to_drive_2016_amended_Aug2017.pdf [Accessed 18 Sep 2019].

3 National highway traffic safety administration. Driver fitness medical guidelines. United States of America, 2009. Available: https://www. nhtsa.gov/sites/nhtsa.dot.gov/files/811210.pdf [Accessed 3 Oct 2019].

4 Determining Driver Fitness in Canada. Canada. Canadian council of motor transport administrators, 2020. Available: https://www.ccmta. ca/images/pdf-documents-english/dv/NSC_6/National-Safety-CodeStandard-6-Determining-Fitness-to-Drive-in-Canada-January-2020. pdf [Accessed 5 Dec 2020].

5 Medical aspects of fitness to drive. New Zealand. New Zealand transport agency, 2014. Available: www.nzta.govt.nz/resources/ medical-aspects/ [Accessed 3 Oct 2019].

6 Engel K, Huckhagel T, Gulberti A, et al. Towards unambiguous reporting of complications related to deep brain stimulation surgery: a retrospective single-center analysis and systematic review of the literature. PLoS One 2018;13:e0198529.

7 Chen T, Mirzadeh Z, Chapple K, et al. Complication rates, lengths of stay, and readmission rates in "awake" and "asleep" deep brain simulation. J Neurosurg 2017;127:360-9 https://search.ebscohost. com/login.aspx?direct=true\&AuthType $=$ ip, athens $\& \mathrm{db}=$ edo\&AN= 124367667\&site=eds-live

8 Fernández-Pajarín G, Sesar A, Ares B, et al. Delayed complications of deep brain stimulation: 16-year experience in 249 patients. Acta Neurochir 2017;159:1713-9 https://search.ebscohost.com/login. aspx?direct=true \&AuthType $=$ ip, athens $\& \mathrm{db}=\mathrm{mdc} \& \mathrm{AN}=28646465 \&$ site=eds-live

9 Tonge M, Ackermans L, Kocabicak E, et al. A detailed analysis of intracerebral hemorrhages in DBS surgeries. Clin Neurol Neurosurg 2015;139:183-7 https://search.ebscohost.com/login.aspx?direct= true\&AuthType $=i p$, athens $\& d b=$ edselp\&AN=S0303846715300482\& site=eds-live

10 Levi V, Carrabba G, Rampini P, et al. "Short term surgical complications after subthalamic deep brain stimulation for Parkinson's disease: does old age matter?". BMC Geriatr 2015;15:116 https://search.ebscohost.com/login.aspx?direct=true\& AuthType $=i p$, athens $\& d b=$ dc $\& A N=26438346 \&$ site $=$ eds-live

11 Verla T, Marky A, Farber $\mathrm{H}$, et al. Impact of advancing age on post-operative complications of deep brain stimulation surgery for essential tremor. J Clin Neurosci 2015;22:872-6 https://search. ebscohost.com/login.aspx?direct=true\&AuthType=ip, athens $\& \mathrm{db}=$ edselp\&AN=S0967586814006833\&site=eds-live

12 Fenoy AJ, Simpson RK. Risks of common complications in deep brain stimulation surgery: management and avoidance. J Neurosurg 2014;120:132-9 https://search.ebscohost.com/login.aspx?direct= true\&AuthType $=$ ip, athens\&db=edo\&AN=93404552\&site=eds-live

13 Doshi PK. Long-term surgical and hardware-related complications of deep brain stimulation. Stereotact Funct Neurosurg 2011;89:89-95 https://search.ebscohost.com/login.aspx?direct=true\&AuthType=ip, athens $\& \mathrm{db}=\mathrm{mdc} \& \mathrm{AN}=21293168 \&$ site=eds-live

14 Voges J, Waerzeggers Y, Maarouf M, et al. Deep-brain stimulation: long-term analysis of complications caused by hardware and surgery-experiences from a single centre. J Neurol Neurosurg Psychiatry 2006;77:868-72 https://search.ebscohost.com/login. aspx?direct=true\&AuthType $=$ ip, athens $\& d b=p s y h \& A N=2006-08588-$ $007 \&$ site $=$ eds-live
15 Goodman RR, Kim B, McClelland S, et al. Operative techniques and morbidity with subthalamic nucleus deep brain stimulation in 100 consecutive patients with advanced Parkinson's disease. J Neurol Neurosurg Psychiatry 2006;77:12-17 https://search.ebscohost. com/login.aspx?direct=true\&AuthType=ip, athens $\& \mathrm{db}=\mathrm{mdc} \& A \mathrm{AN}=$ 16361585\&site=eds-live

16 Kocabicak E, Temel Y. Deep brain stimulation of the subthalamic nucleus in Parkinson's disease: surgical technique, tips, tricks and complications. Clin Neurol Neurosurg 2013;115:2318-23 https:// search.ebscohost.com/login.aspx?direct=true\&AuthType=ip,athens\& $\mathrm{db}=\mathrm{mdc} \& \mathrm{AN}=24041965$ \& site $=$ eds-live

17 Rughani Al, Hodaie M, Lozano AM. Acute complications of movement disorders surgery: effects of age and comorbidities. Mov Disord 2013;28:1661-7 https://search.ebscohost.com/login.aspx?

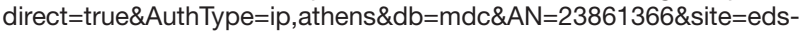
live

18 Coley E, Farhadi R, Lewis S, et al. The incidence of seizures following deep brain stimulating electrode implantation for movement disorders, pain and psychiatric conditions. $\mathrm{Br} J$ Neurosurg 2009;23:179-83 https://search.ebscohost.com/login.aspx?direct= true\&AuthType $=i p$, athens\&db=mdc\&AN=19306174\&site=eds-live

19 Pouratian N, Reames DL, Frysinger R, et al. Comprehensive analysis of risk factors for seizures after deep brain stimulation surgery. $J$ Neurosurg 2011;115:310-5 https://search.ebscohost.com/login. aspx? direct=true \&AuthType $=\mathrm{ip}$, athens $\& \mathrm{db}=\mathrm{edo} \& \mathrm{AN}=65052418 \&$ site=eds-live doi:10.3171/2011.4.JNS102075

20 Carlson JD, Neumiller JJ, Swain LDW, et al. Postoperative delirium in Parkinson's disease patients following deep brain stimulation surgery. J Clin Neurosci 2014;21:1192-5 https://search.ebscohost. com/login.aspx?direct=true\&AuthType $=$ ip, athens\& $\mathrm{db}=$ edselp\&AN $=$ S0967586813006875\&site=eds-live

21 Wang $X-Q$, Zhuang $\mathrm{H}-\mathrm{X}$, Zhang L-X, et al. Nomogram for predicting postoperative delirium after deep brain stimulation surgery for Parkinson's disease. World Neurosurg 2019;130:e551-7 https:// search.ebscohost.com/login.aspx?direct=true\&AuthType=ip,athens\& $\mathrm{db}=$ edselp\&AN=S1878875019318005\&site=eds-live

22 Tanaka M, Tani N, Maruo T, et al. Risk factors for postoperative delirium after deep brain stimulation surgery for Parkinson disease. World Neurosurg 2018;114:e518-23 https://search.ebscohost. com/login.aspx?direct=true\&AuthType=ip, athens\&db=edselp\&AN= S1878875018304911\&site=eds-live

23 Witt K, Daniels C, Volkmann J. Factors associated with neuropsychiatric side effects after STN-DBS in Parkinson's disease. Parkinsonism Relat Disord 2012;18:S168-70 https://search. ebscohost.com/login.aspx?direct=true\&AuthType=ip, athens $\& \mathrm{db}=$ edselp\&AN=S1353802011700529\&site=eds-live

24 Witt K, Daniels C, Reiff J, et al. Neuropsychological and psychiatric changes after deep brain stimulation for Parkinson's disease: a randomised, multicentre study. Lancet Neurol 2008;7:605-14 https:// search.ebscohost.com/login.aspx?direct=true\&AuthType=ip, athens\& $\mathrm{db}=\mathrm{mdc} \& \mathrm{AN}=18538636 \&$ site $=$ eds-live

25 Cyron D. Mental side effects of deep brain stimulation (DBS) for movement disorders: the futility of denial. Front Integr Neurosci 2016;10:17 https://search.ebscohost.com/login.aspx?direct=true\& AuthType $=$ ip, athens \&db=psyh\&AN=2016-29791-001\&site=eds-live

26 Buhmann C, Vettorazzi E, Oehlwein C. Impact of deep brain stimulation on daily routine driving practice in patients with Parkinson's disease. Solvay Pharmaceut 2015 https://search. ebscohost.com/login.aspx?direct=true\&AuthType=ip, athens\&db= edsdoj\&AN=edsdoj.9ce51b2c6c8d47f9a1bd797f77c01242\&site= eds-live

27 Buhmann C, Maintz L, Hierling J, et al. Effect of subthalamic nucleus deep brain stimulation on driving in Parkinson disease. Neurology 2014;82:32-40 https://n.neurology.org/content/82/1/32

28 Yin Z, Bai Y, Guan B, et al. A quantitative analysis of the effect of bilateral subthalamic nucleus-deep brain stimulation on subjective and objective sleep parameters in Parkinson's disease. Sleep Med 2021;79:195-204 https://search.ebscohost.com/login.aspx?direct= true\&AuthType $=i p$, athens $\& d b=m d c \& A N=33208282 \&$ site $=$ eds-live

29 Uitti RJ. Parkinson's disease and issues related to driving. Parkinsonism Relat Disord 2009;15:S122-5.

30 Lloyd K, Gaunt D, Haunton V, et al. Driving in Parkinson's disease: a retrospective study of driving and mobility assessments. Age Ageing 2020;49:1097-101.

31 van den Dool J, Visser B, Huitema RB, et al. Driving performance in patients with idiopathic cervical dystonia; a driving simulator pilot study. Front Neurol 2020;11:229 https://www.frontiersin.org/articles/ 10.3389/fneur.2020.00229/full 